\section{Nuclear Cardiology Technology Study Guide}

\author{
S.G. Johnson, M.B. Farrell, A.M. Alessi, and M.C. Hyun
}

Reston, VA: Society of Nuclear Medicine and Molecular Imaging, 2015, 210 pages, $\$ 199$

This second edition of Nuclear Cardiology Technology Study Guide offers a complete study of nuclear cardiology from basic cardiac anatomy to advanced processing and quantitative analysis. The material is presented in a logical sequence, following the content specifications of the NMTCB nuclear cardiology specialty examination, with many complex topics addressed and explained in a way that is easy for students to understand. It begins by augmenting the reader's fundamental knowledge of anatomy, pathophysiology, and electrocardiography, beginning with a discussion of first-pass, gated wall motion, and shunt studies before progressing to myocardial perfusion studies. Each topic relating to myocardial perfusion is simplified into manageable sections that include indications and contraindications, patient preparation, radiopharmaceuticals, stress protocols, acquisition protocols, processing techniques, quality control, image artifacts, and case reviews. SPECT, SPECT/CT, and PET are included in this discussion.

Chapter 1 serves as a good introduction to topics discussed in the rest of the book. A complete review of the structure and function of the heart, coronary arteries, conduction system, and common diseases is provided, as well as an explanation of cardiac catheterization. Chapter 2 expands on the electrical conduction of the heart, covering proper placement of electrocardiogram leads, explanations of each section of an electrocardiogram waveform, and the steps to interpreting electrocardiograms. Also included are high-quality images of various arrhythmias, with detailed text on the cause of the abnormality and how to recognize it on an electrocardiogram.

Chapter 3, a useful review of nonmyocardial perfusion cardiovascular examinations, covers equilibrium radionuclide angiography, first-pass angiography, left-to-right shunts, right-to-left shunts, myocardial infarction, myocardial viability, metaiodobenzylguanidine studies, and ${ }^{123}$ I-iodofiltic acid studies. Each type of study reviewed includes patient preparation, the radiopharmaceuticals, data acquisition, patient positioning, processing, and associated mathematic formulas. High-quality procedural images are included.

Chapter 4 covers all the components needed to acquire highquality non-PET myocardial perfusion images: the indication, the patient preparation, and the data acquisition. The useful tables effectively summarize medications, beverages, and foods that must be discontinued before the procedure. Differences in protocols are explained, including 2-d technetium, 1-d rest/stress, 1-d stress/rest, dual-isotope, ${ }^{201} \mathrm{Tl}$ distribution, and gating protocols, with a chart summarizing the information. Other topics include appropriate injection-to-scan times, patient positioning, pixel size, energy window settings, camera orbits, collimators, projection and orbit selections, gating, and attenuation correction. The chapter ends with explana-

COPYRIGHT (c) 2016 by the Society of Nuclear Medicine and Molecular Imaging, Inc. tions of myocardium stunning verses hibernation and transient ischemic dilation values, along with clear, full-color images of each.

Beginning with an advantageous review of the physics of PET, chapter 5 focuses on the physics and radiopharmaceuticals used in myocardial PET imaging. Spatial resolution and attenuation correction are included, as well as a breakdown of myocardial perfusion and myocardial metabolism imaging tracers.

Chapter 6 comprehensively reviews cardiac medications and common medical emergencies a technologist may encounter in a cardiac stress laboratory. Tables are used to summarize drug classes, their uses, and their mechanisms of action. The medications discussed include angiotensin-converting enzyme inhibitors, antiarrhythmics, anticoagulants, antiplatelet drugs, $\beta$-blockers, calcium channel blockers, antilipemics, cardiac glycosides, diuretics, sympathomimetic drugs, and vasodilators. The most life-threatening and most common medical emergencies are explained, along with tips on recognizing a medical emergency effectively and appropriately managing it.

Chapter 7 provides a complete overview of non-PET radiopharmaceuticals currently used for myocardial imaging. The discussion of each radiopharmaceutical includes its unique preparation guidelines, contraindications, mechanism of action, biodistribution, and recommended dosage, as well as tips on imaging with it.

Chapter 8 , on processing techniques, begins with general principles and acquisition considerations before moving on to more complex techniques. The use of filtered backprojection and iterative reconstruction is reviewed, and a detailed yet simple and easy-tounderstand explanation is given of filters, including considerations in choosing the appropriate filter. Also provided is an overview of orientation and nomenclature, analysis of processed data, and interpretation of quantitative data.

Chapter 9 explains the 3-part integrated analysis of quantification applicable to myocardial perfusion imaging, beginning with an overview of commercially available software presented in such a way as to make the subject easy for the reader to understand. Some of the topics covered are 17- and 20-segment score systems, myocardial wall motion and thickening, diastolic function, phase analysis, lung-to-heart ratio, transient ischemic dilation, quantitation of myocardial blood flow, and sources of technical error.

It is important for a responsible and competent technologist to understand the appropriate use of nuclear cardiology studies as well as the elements needed to accurately interpret and report the data provided. Chapter 10 helps the reader achieve this understanding by providing a simple description of image interpretation and reporting and a step-by-step process. The chapter ends with an overview of appropriate-use criteria, as well as a simple how-to guide.

Chapter 11 is a detailed survey of quality control procedures for equipment and the importance of following a well-designed quality assurance program. An overview and simple explanation of quality control procedures for SPECT, SPECT/thermoacoustic CT, SPECT/CT, and nonimaging instruments is provided, as well as their relative importance and the frequency with which they should be performed.

Chapter 12 begins by reviewing the important concepts associated with exercise stress testing. Patient preparation, various exercise protocols, safety risks, calculation of maximum predicted and target 
heart rates, and considerations for special patient populations are all discussed in depth. Then, in chapter 13, virtually all aspects of pharmacologic stress agents are discussed, including the indications for a pharmacologic examination, patient preparation, dosage, mechanism of action, administration protocol, common side effects, contraindications, and anecdotes on each agent.

Chapter 14 is on risk stratification and patient management. The manifestations of ischemia are reviewed, the diagnostic accuracy of various noninvasive examinations is described, the concept of risk identification is introduced, and the important role that myocardial perfusion imaging plays in the accuracy of risk assessment and patient management is detailed.

The last chapter thoroughly discusses dosing, image acquisition time, patient positioning, contamination, patient motion, gating, arrhythmias, attenuation, diseases, equipment, and processing-related artifacts. A plethora of images is included, helping the reader both to understand and then to apply the information.

This full, comprehensive book includes many useful tables and color images that, besides helping the reader understand the material, can serve as a quick reference. Ten focused review questions are provided at the end of each chapter, and a 125-question mock examination concludes the book.

Nuclear Cardiology Technology Study Guide is the perfect text for educators, students, residents, physicians, or anyone seeking a greater understanding of nuclear cardiology. This book should be the go-to resource for technologists studying for the NMTCB nuclear cardiology specialty examination.

Sara Johnson, CNMT 4001 W. Tampa Bay Blvd. Hillsborough Community College Tampa, FL 33614 E-mail: sjohnson215@hccfledu 